\title{
VERS UNE NOUVELLE DÉMOCRATIE ? UN NOUVEAU SYSTÈME...
}

\author{
Author(s) / Auteur(s) : \\ Eva-Marie BRUNO \\ Étudiante en études européennes à l'Institut des études européennes à Bruxelles (ULB) \\ eva02brune@hotmail.com
}

\begin{abstract}
Résumé :
L'avènement de la démocratie dans nos régions occidentales est le fruit d'un long processus historique. De nombreux facteurs ont permis son développement. Commençons par un facteur fondamental : la sécularisation progressive 'la religion en dehors des affaires de l'Etat'. Une tendance à restreindre le culte au cercle uniquement privé, voir individuel. Penchons-nous sur ce critère de l'individualisme : l'égalité des chances en voilà un précepte révolutionnaire. Donner la possibilité à chacun de choisir son parcours de vie. Un autre facteur important : le développement d'un système économique de référence : le capitalisme, précurseur de l'avènement de l'Etat providence. L'Etat devient garant de la sécurité financière et médicale de ses citoyens. Ce système fut instauré, au lendemain de la seconde guerre mondiale. Jamais dans l'histoire des Etats-nations européens, les nations avaient été aussi homogènes. Ces nations homogènes changèrent rapidement pour laisser place à une immigration de masse et la création d'une société multiculturelle. Actuellement, de nombreuses tensions se font sentir, il semblerait que le 'vivre ensemble' est en crise. Une véritable bombe à retardement. Pourquoi ne pas créer un nouveau système pour compléter celui d'Etat-providence ? Et pourquoi pas une démocratie culturelle avec la mise en avant de préceptes, règles fondamentales déjà existantes : règles de savoir vivre, codes tacites, règles de moeurs jamais explicitement énoncées. Si chaque citoyen prenait connaissance de ces règles et les appliquait, cela pourrait mener à une meilleure cohésion sociale sur nos terres démocratiques.
\end{abstract}

\section{Keywords / Mots-clés :}

démocratie, états/nations, culture, normes, changement et citoyenneté

L'avènement de la démocratie dans nos régions occidentales est le fruit d'un long processus historique. De nombreux facteurs ont permis son développement. Commençons par un facteur fondamental : la sécularisation progressive la religion en dehors des affaires de l'Etat. Une tendance à restreindre le culte au cercle uniquement privé, voir individuel. Penchons-nous sur ce critère de l'individualisme : l'égalité des chances en voilà un précepte révolutionnaire. Donner la possibilité à chacun de choisir son parcours de vie. Un autre facteur important : le développement d'un système économique de référence : le capitalisme, précurseur de l'avènement de l'Etat providence. L'Etat devient garant de la sécurité financière et médicale de ses citoyens. Ce système fut instauré, au lendemain de la seconde guerre mondiale. Jamais dans l'histoire des Etats-nations européens, les nations avaient été aussi homogènes. Ces nations homogènes changèrent rapidement pour laisser place à une immigration de masse et la création d'une société multiculturelle. Actuellement, de nombreuses tensions se font sentir, il semblerait que le vivre ensemble est en crise. Une véritable bombe à retardement. Pourquoi ne pas créer un nouveau système pour compléter celui d'Etat-providence ? Et pourquoi pas une démocratie culturelle avec la mise en avant de préceptes, règles fondamentales déjà existantes : règles de savoir vivre, codes tacites, règles de moeurs jamais explicitement énoncées. Si chaque citoyen prenait connaissance de ces règles et les appliquait, cela pourrait mener à une meilleure cohésion sociale sur nos terres démocratiques.

L'instauration de la démocratie en vigueur dans nos régions actuellement est un long processus historique. Les prémisses se situent aux alentours du 15ème siècle et se développent pour laisser place à l'élément déclencheur : la révolution française et ses déploiements constants jusqu'à nos jours. La période contemporaine dans laquelle nous vivons actuellement débute en 1789. Il semblerait que chaque évènement historique, changement parvenu depuis cette période a un impact sur notre mode de vie actuelle. Le changement complet d'une société semble être indispensable pour l'établissement 
d'une démocratie. Afin d'aspirer et d'établir une démocratie représentative dans un Etat, la société doit au préalable se modifier complétement en vue d'appliquer les préceptes de ce régime. En effet, le bon fonctionnement d'une démocratie dépend de ses citoyens. Analysons cela à travers L'histoire des temps modernes qui a permit le développement d'une nouvelle vision de l'homme, la création d'un citoyen.

"L'époque des temps modernes fin du $\mathrm{XV}^{\mathrm{e}}$ siècle jusqu'à la révolution française est marquée par la maîtrise croissante de l'homme sur le monde et sur son propre destin. Elle a préparé le libéralisme et la démocratie parlementaire, aboutissant à une société laïcisée, à une nouvelle anthropologie ainsi qu'une nouvelle conception de l'homme qui est celle du monde dans lequel chaque individu est censé faire ses choix et ses traits particuliers de la société occidentale que d'être individualiste." (Bernard, 2014, p.1)

À travers cet extrait, on peut observer les apports de cette période : l'homme choisit son propre destin et peut faire ses propres choix, la mise en avant de l'individualisme et l'aboutissement vers une société laïcisée. Concernant ce principe de laïcité : Le philosophe John Locke (1632-1704), dans ses lettres de tolérance 1689-92, justifiait ce concept de tolérance, un précepte fort pour la démocratie. Celle-ci favorise la vie en société, la religion se limite donc se limiter au domaine privé : le spirituel doit être séparé de temporel (Bernard, 2014, p.166).

La révolution française et la déclaration universelle des droits de l'homme et du citoyen du 26 août 1789 marque un tournant dans l'histoire (Bernard, 2014, p.189) :

- "Les hommes naissent et demeurent libres et égaux en droits"

- "droits à la liberté (sans nuire à autrui) de presse et de culte, propriété, sûreté et la résistance à l'oppression"

- "le principe de souveraineté réside essentiellement dans la Nation"

- "tout homme est présumé innocent jusqu'à ce qu'il ait été déclaré coupable"

La vision d'un homme nouveau voit le jour pendant la période moderne. La révolution française et l'époque contemporaine vont laisser place à la création d'une société nouvelle et la création d'un Etatnation. Le citoyen est partie intégrante d'une nation. Tous ces préceptes sont encore d'actualité de nos jours.

En ce qui concerne le développement du capitalisme, il s'est essentiellement développé au sein d'Etats où les préceptes propres à l'élaboration ou l'installation d'un régime de démocratie représentative étaient de vigueur. Selon Max Weber, les innovations économiques étaient reliés à d'autres aspects propres de la civilisation occidentale tels que la notion de l'Etat au sens moderne, le droit rationnel, le concept de citoyen, la science au sens moderne, l'ethos rationnel de la conduite de la vie, (il s'agit d'une conduite de vie qui refuse toute oisiveté, où chaque instant, chaque acte doit être à tout moment rentabilisé) (Vogel, 2013, pp.47-48). Ces préceptes propres à un régime démocratique sont des facteurs qui ont permis le développement du capitalisme dans nos régions.

À la fin de la seconde guerre mondiale un nouveau type d'Etat, un nouveau système voit le jour. Il s'agit de l'Etat providence : un Etat créance, responsable de la prise en charge de ses citoyens au moyens d'allocations, soutiens financiers, aides aux personnes. Cette notion d'Etat providence à une dimension universelle. L'Etat de créance est valable pour tous. Ce système fût instauré dans un contexte d'après-guerre, de reconstruction de l'unité nationale. Cette logique de partage à permit de renforcer les capacités économiques de chacun afin de permettre une certaine autonomie et sécurité. Un nouveau type d'Etat se développe depuis quelques temps, il s'agit de l'Etat réflexif, celui-ci met en avant l'autonomisation des personnes et le renforcement de leurs capacités. Tout un chacun doit mettre en avant ses revendications, investir, développer une relation positive aux personnes. Avoir une dimension socio-relationnelle. Ce type de comportement reste très rare dans les faits. Devrions-nous le compléter cet Etat réflexif à l'aide d'une éducation citoyenne pour tous dès le plus jeune âge (Cantelli, 2016)?

La démocratie représentative est en crise dans nos régions et largement critiquée par ses citoyens. Pourquoi certains citoyens se laissent séduire par des partis populistes, voire d'extrême droite. "Un mouvement extrémiste repose sur l'affirmation que les mécanismes démocratiques traditionnels sont 
inefficaces pour les objectifs qu'il poursuit, et que par conséquent il faut employer des méthodes capables de briser le cadre démocratique." (Finley, 2003, p.122)

L'intérêt national est nourri par la croissance économique. Lorsqu'une grosse entreprise ferme, les conséquences sont immédiates sur le plan du chômage, du niveau de vie, les inégalités au sein de la société. Actuellement dans les pays développés, la croissance devient très lente et il est envisageable que l'on se situe dans une crise rampante (Vogel, 2013, p.76). Ce taux de croissance semble chuter d'années en années. Cette situation précaire est néfaste pour la société (Vogel, 2013, p.87). Des citoyens démunis se sentent délaissés par leurs gouvernements et se tournent vers des partis politiques populistes qui adaptent leurs discours par rapport aux attentes des citoyens.

"La conviction que la démocratie est la meilleure forme d'organisation politique" (Vogel, 2013, p.55) . Comment convaincre les citoyens que la démocratie représentative est la meilleure forme de régime envisageable ? Faudrait-il éduquer les citoyens à avoir une conscience politique, à travers des leçons d'histoires, de pensée politique. Former les citoyens aux fondements de la démocratie et des valeurs propres à ce système.

Voici l'exemple d'un précepte démocratique : la notion de droits et de devoirs de chaque citoyen.

"Attribuer un droit à quelqu'un signifie lui reconnaitre la faculté de faire ou ne pas faire quelque chose à son gré, et simultanément le pouvoir de résister, en recourant en dernière instance également à sa propre force ou à celle des autres, contre l'agresseur éventuel, lequel a par conséquent le devoir (ou l'obligation) de s'abstenir de tout acte qui puisse d'une manière ou d'une autre interférer avec cette faculté de faire ou de ne pas faire [...] Droit et devoir sont deux notions appartenant au langage prescriptif, en tant que celles-ci présupposent l'existence d'une norme ou règle de conduite qui, à partir du moment où elle attribue à un sujet la faculté de faire ou de ne pas faire quelque chose, impose à quiconque de s'abstenir de toute action susceptible d'une façon ou d'une autre d'empêcher l'exercice de cette faculté." (Bobbio, 1996, pp.15-16)

Ce précepte est un dérivé d'une loi naturelle, il s'agit d'une conception générale et hypothétique de la nature humaine dont la connaissance parfois non-connue mène à des troubles dans la société.

Un autre extrait issu d'un autre ouvrage souligne la notion de liberté et ses limites. Selon John Stuart Mill dans l'introduction de son livre La liberté, il cite :

"Le seul objet qui autorise les hommes individuellement ou collectivement, à troubler la liberté d'action d'aucuns de leurs semblables, est la protection de soi-même. La seule raison légitime que puisse avoir une communauté pour user de force contre un de ses membres est de l'empêcher de nuire aux autres." (Finley, 2003, p.125)

Une société idéale, serait une société au sein de laquelle, chaque citoyen est libre de faire ses propres choix, de maximaliser au maximum ses ressources. Se respecter l'un l'autre à l'aide de préceptes communs issus de la démocratie.

\section{RÉFÉRENCES}

BERNARD, Bruno (2014). Histoire des temps modernes. Bruxelles : Presses de l'Université libre de Bruxelles.

BOBBIO, Noberto (1996). Libéralisme et démocratie. Paris : Cerf.

CANTELLI, Fabrizio (2016). Politiques publiques. Notes de cours, avril 2016.

FINLEY, Moses (2003). Démocratie antique et démocratie moderne. Paris : Payot \& Rivages.

VOGEL, Jean (2013). Transformation des systèmes économiques et sociaux depuis le XVIII siècle. Bruxelles : Presses de l'Université libre de Bruxelles. 
